\title{
Investigation of Deep Convolutional Neural Network (CNN) approaches' accuracy for the detection of COVID-19
}

\author{
Esraa Fady Dawood ${ }^{1}$, Nader Mahmoud ${ }^{2}$ and Ashraf Elsisi ${ }^{3}$ \\ Department of Computer Science (CS), Faculty of Computers and Information \\ Menoufia University, Egypt \\ 1esraa_fady@yahoo.com, ${ }^{2}$ nader.mahmoud@ci.menofia.edu.eg, ${ }^{3}$ ashraf.elsisi@ci.menofia.edu.eg
}

\begin{abstract}
- the world those days focuses on protecting human health and combating the irruption of coronavirus patients (COVID-19). As results of its extra ordinarily contagious infection that have caused a disturbance in everyone's lives in various ways. For early screening, Reverse Transcription Protein Chain Reaction (RT-PCR) test is used to examine the onset of the patients by detecting the RNA material of the virus among the patients' samples. Recent results indicate that the applying of $X$-ray images and $X$-radiation (CT) improves the detection accuracy of this disease. However, the classification task of medical images is tough due to several factors such as lack of dataset for COVID-19, and difficulty in identifying type of infection. Recent research works have been proposed for COVID-19 detection that has been applied on specific datasets. Thus, it is vital to validate their performance on various datasets with different imaging disease conditions. The paper presents a comparison study between top performer CNN models that recorded the very best detection accuracy in image detection and classification: COVID-Net, VGG16, ResNet, Bayesian, DenseNet, and DarkNet. Such CNN approaches can assist medical staff in the early detection of infection. Additionally, we improved dataset in terms of quality, clarity, and quantity using augmentation technique. The quantitative results show that Darknet and COVID-net yield high detection accuracy when applied on CT and X-ray dataset. We validated our results by training the models on multiple different datasets, using CPU and GPU with various bach sizes and optimizers.
\end{abstract}

Keywords-COVID-19, Computerized Tomography, Chest X-ray, CNN, Deep Learning.

\section{INTRODUCTION}

Recently, COVID-19 virus has created a tremendous miss all over the world. As a result, a fast and reliable scanning tool is warranted to detect those patients who have been infected with this virus. Currently, RT-PCR is considered as the primary used tool for testing COVID-19 patients by detecting the virus samples collected from the mucus of the upper respiratory tract of those patients [1]. Nevertheless, being in high demand made RT-PCR a time consuming tool as it takes time for one to be tested, leading to further delay in the result. Consequently, recent research works have studied the possibility of using traditional imaging tools a quick alternative for virus detection for the detection of the virus [2]. CT scan is in an exceedingly position to supply elaborate 3D images of internal anatomical structures like organs, bones, soft tissues, and blood vessels. It consists of a set of slices for a particular area of the body whereas not overlapping varied body structures [3]. These images area unit conversant in facilitate in various medical interventions, e.g: magnified Reality overlay in minimally invasive surgery [4], A Multitier Deep Learning Model for Arrhythmia Detection [5]. X-rays produce different type of images, once passing through body, bone and different dense objects block the radiation and look white on the X-ray scan. The less-dense tissues area unit arduous to be visible and so appears grey.

Convolutional neural network $(\mathrm{CNN})$ is utilized in computer vision for image content analysis. However, the classification of medical images classification of medical images is challenging due to: poor image quality, and difficult lighting condition, i.e, poor image quality, and hard lighting conditions. The pre-trained deep CNN models have shown good potential to extract COVID-19's specific graphical features to reduce time for disease identification [6]. Several research works have been proposed for COVID-19 detection that yielded encouraging detection accuracy. However, these research works are only evaluated and validated on specific datasets. 
In this paper, we present a comparison study between top-performer CNN models. We include in the comparison different dataset of both $\mathrm{CT}$ and X-ray images with various size and conditions. And achieving high accuracy up to 0.99 with the COVID-Net model on the CT dataset and 0.98 with the DarkNet model on the $\mathrm{x}$-ray dataset. This comparison study is crucial to direct future analysis on the proper path.

The paper is divided into the following sections: Section II, which discusses the related works of the six models and a comparison of their advantages and disadvantages. Section III, presents the experimental results of the six CNN model of the study. We applied three phases of comparison and on different datasets. Conclusion is discussed in Section IV.

\section{RELATED WORKS}

CNN has associate prestigious history in pc vision. It has been strongly competitive with other alternative ways for recognition and classification purposes. Recently, CNN showed better performance in the field of large-scale visual recognition. This in turn, made the researchers more motivated to pre-trained CNNs adapted to alternative domains and datasets [7]. Moreover, CNN will yield an additional discriminative illustration of image, which is crucial for the fine grained image classification.

Typical CNN is presented below in Fig. 1. It has many convolutional layers, pursued by pooled layers and a group of fully connected layers within the conclusion. Those layers among CNNs are organized in 3 dimensions with the inputs $(x), m \times m \times r$, wherever $m$ refers to the height and width of the input and $r$ refers to the depth or the channel numbers. Many filters called kernels $(K)$ of size $n \times n \times q$ are in every convolutional layer.

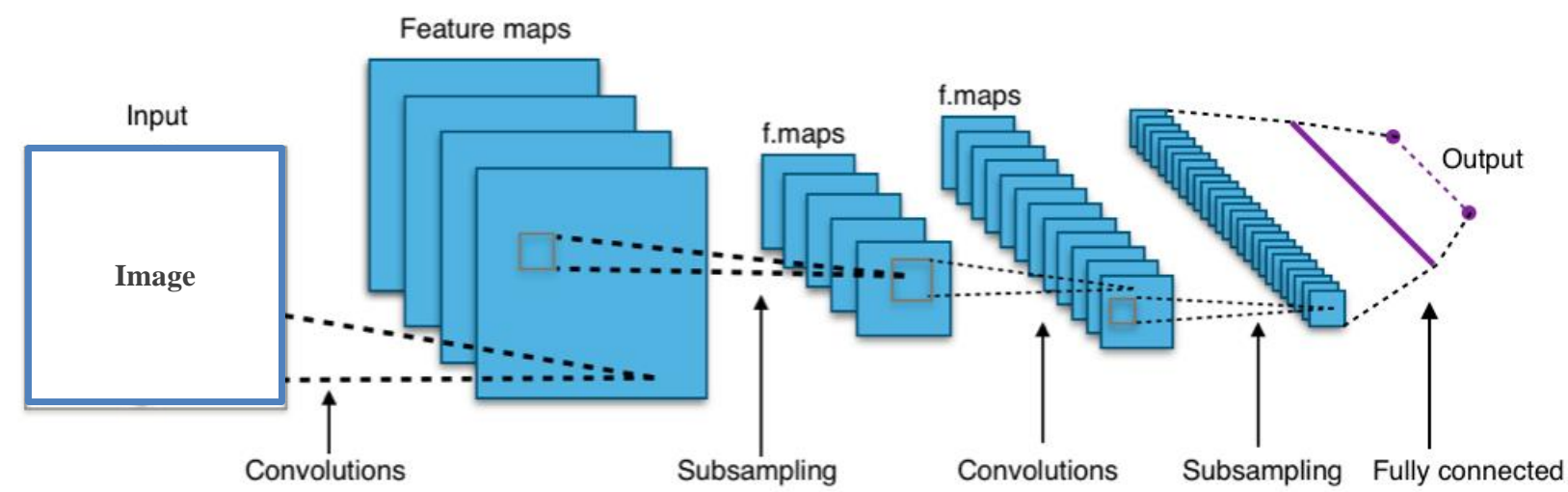

Fig. 1. The architecture of the Convolutional Neural Network (CNN) [7]

Here, $n$ must be less than the scale of the input image dimensions, $q$ is often either smaller or constant size as $r$. The filters are the principle of local connections that are convolved with the input. It shares the same parameters to generate $K$ feature maps, each of size $m-n-1$. In every single feature, there is down sampling to decrease the parameters within the network, fasts the training process, and thus controls over fitting. Pooling operation (either average or max) is completed over a $p \times p$ ( $p$ is that the filter size) contiguous region for all feature maps. In the final stage layers area unit, they are totally connected. These layers take previous low-level and midlevel options and produce a high-level abstraction from both data and classification [8].

Careful discussion for deep learning models for COVID-19 identification as COVID-Net, VGG16, ResNet, Bayesian, DenseNet, and DarkNet within the next sub-sections.

\section{A. Bayesian [9]}

Convolutional encoder-decoder network (or Bayesian) is employed as core segmentation engine within brain extraction works flow as shown in Fig. 2. This network consists of an associate encoder network and reversed decoder network. Encoder network produces a function of feature extraction and data compression, whereas decoder network gathers compressed features to the original image size victimization extracted options via multi-scale distributed upsampling. Networks of encoders and decoders were made employing a series of convolutional layers, 
batch social control, ReLU non-linearity, and most pooling layers or upsampling layers. Tabel I, shows the strongest and weakness points of Bayesian model.

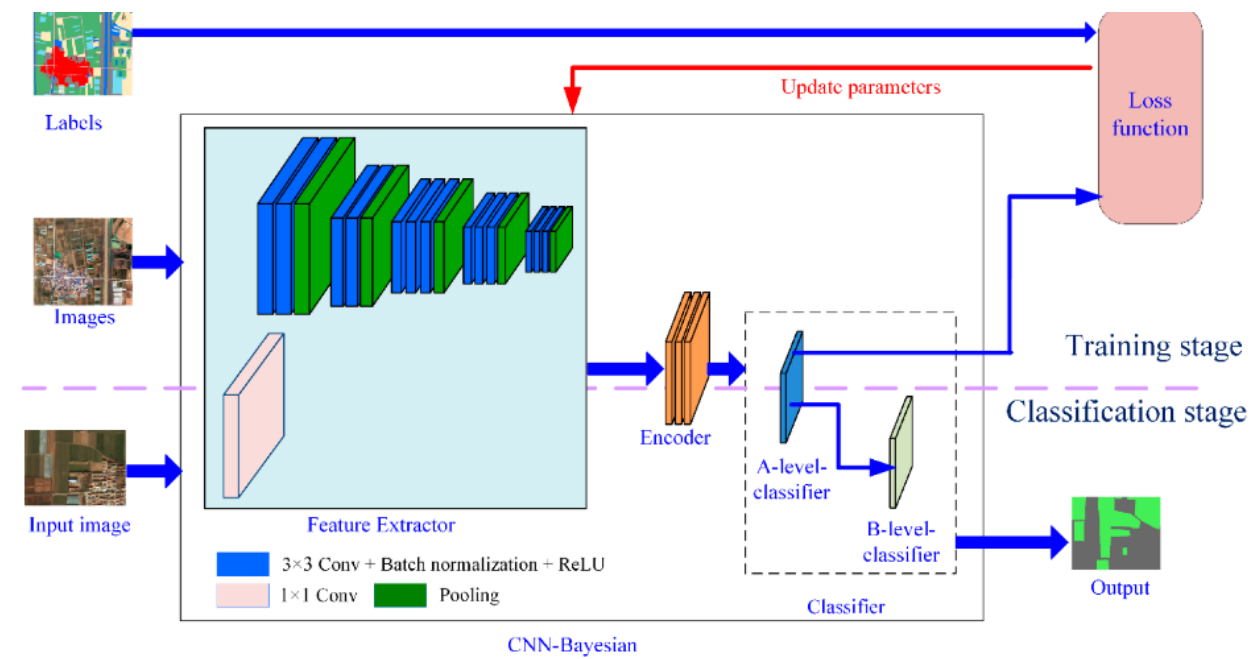

\section{B. DenseNet [12]}

Fig. 2. The structure of the Bayesian Network [9]

Historically, neural networks need smart coaching, use of feature maps to cut back the time of convolution and sub-sampling processes. As a result, losses happen within the image feature within the transition between layers. In DenseNet each layer is directly connected to any or all alternative layers and every layer has direct access to loss functions and original input signals. Feature-maps of all preceding layers are a unit concatenated and used as input for any partiqular layer, and its feature-maps area unit used as inputs into all subsequent layers.

DenseNet has four dense blocks and transition layers between two consecutive dense blocks as shown in Fig. 3. Every dense block consists of many convolution layers, and every transition layer made up of batch social control, a convolution, and a mean pooling layer. Finally, we've got a totally connected layer with a soft-max activation perform with 3 neurons for three-class classification and two neurons for two-class classification [12]. Tabel I, shows the strongest and weakness points of DenseNet model.

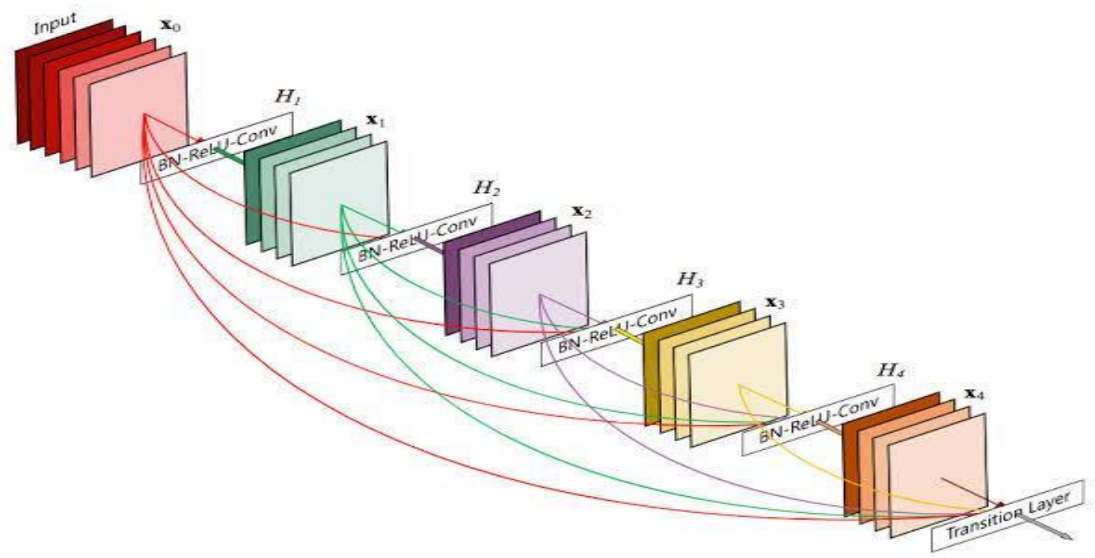

Fig. 3. The structure of the DenseNet architecture [13] 


\section{VGG-16 [14]}

The VGG-16 model was developed with a really tiny convolution within the network. Despite, its simble structure, is completely different than alternative models. Wherever, it provides a lot of in-depth structure that's followed by layers with double or triple convolution layers. The VGG-16 network introduced the thought of grouping multiple convolution layers with smaller kernel sizes rather than having one Convolutional layer with an outsized kernel size.

The VGG-16 design uses 13 convolutional layers and a pair of fully connected layers and one softmax classifier, every of them followed by a Rectified Linear Unit (ReLU) layer and has maximum pooling layers for sampling as shown in Fig. 4 [14]. Its 3 layers that area unit totally connected for classification, 2 of them function hidden layers, whereas ultimate classification layer is made up of a thousand units representing the image classes within the database [15]. Tabel I, shows the strongest and weakness points of VGG model.

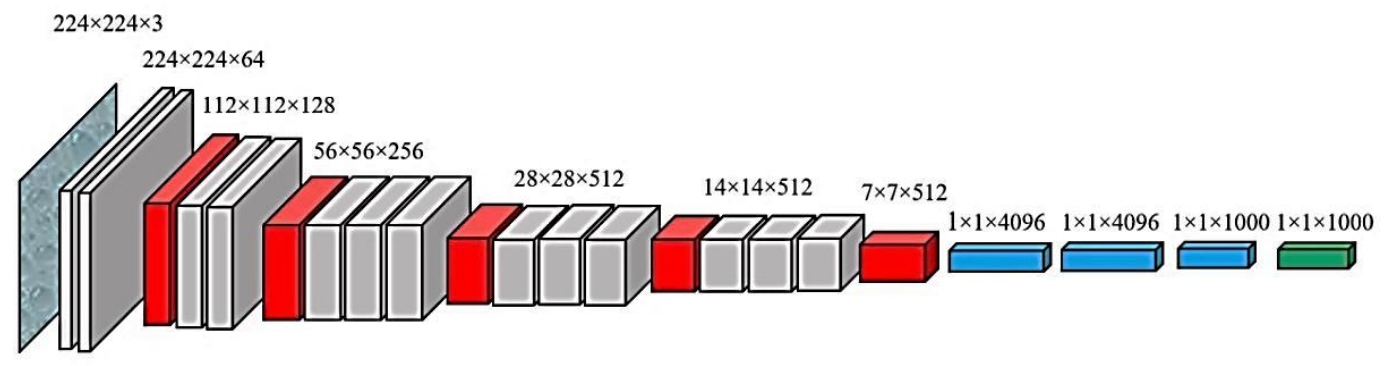

Fig. 4. The structure of the VGG architecture [15]

\section{ResNet [17]}

Previous CNN architectures had a drop off within the effectiveness of additional layers, ResNet will add an outsized variety of layers with a powerful performance. ResNet was associate innovative resolution to the vanishing gradient problem. Neural networks train via the back propagation method, which depends on gradient descent, moving down the loss performs to seek out the weights that deliver the minimization [18]. Having a lot of layers, the perennial multiplication reduces the gradient until it vanishes, inflicting the performance to saturate. As shown in Fig. 5. Presents the design of the residual mapping of this model.

ResNet stacks layers that originally don't do something, skips them, reusing the activations from previous layers. Skipping at the start compresses the network into solely a couple of layers. Then, once the network trains once more, all layers area unit enlarged and therefore the "residual" elements of the network explore a lot of the feature space of the image. Tabel I, shows the strongest and weakness points of ResNet model.

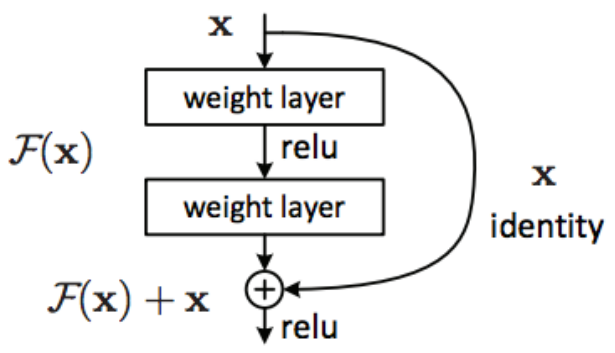

Fig. 5. The architecture of the Residual mapping structure [19] 


\section{E. COVID-Net [20]}

COVID-Net is that the initial neural network designed for the detection of COVID-19 to produce a light-weight projection-expansion-projection-extension (PEPX) design that allows increased representational capability which greatly reducing the complexity of computation. The network made via a machine-driven design exploration strategy that is machine-controlled and shows a high architectural diversity as evident by the heterogeneous composition of conventional spatial convolution layers, point-wise convolutional layers, and depth-wise convolution layers in a manner that strikes a balance between accuracy, architectural and computational complexity. Tabel I, shows the strongest and weakness points of COVID-Net model.

The COVID-Net architecture shown in Fig. 6. Consists of:

1) First stage Projection: $1 * 1$ convolutions for projecting input features to lower dimension,

2) Expansion: $1 * 1$ convolutions for expanding feat to higher dimension that differs than input features,

3) Depth-wise Representation: efficient $3 * 3$ depth wise convolutions, in order to learn spatial characteristics in order to minimize computational complexity while preserving representational capacity,

4) Second-stage Projection: $1 * 1$ convolutions for projecting features back to lower dimension, and

5) Extension: $1 * 1$ convolutions that finally extend channel dimensionality to higher dimension to make final version of the features [20].

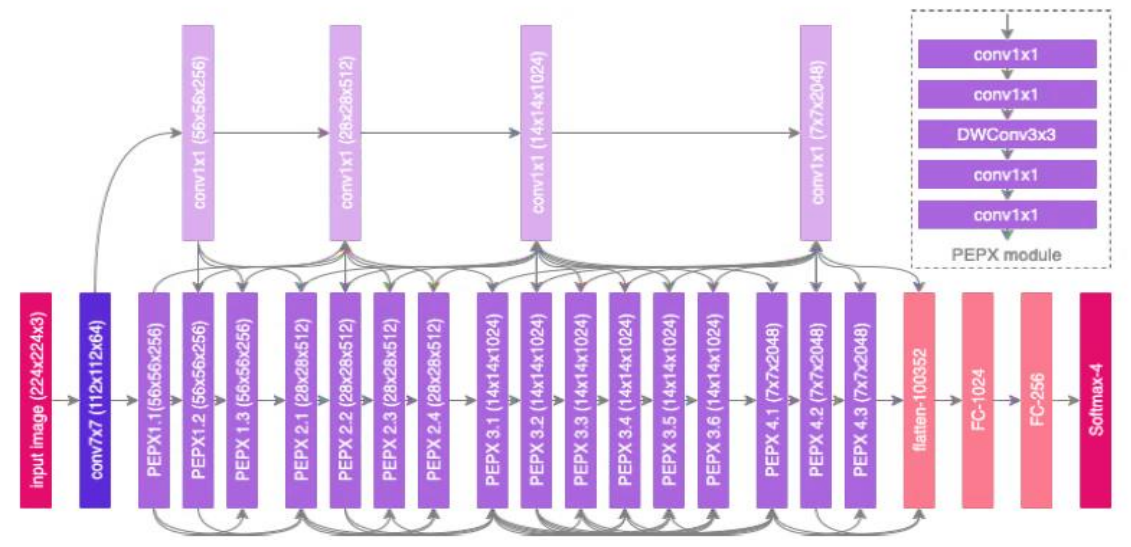

\section{F. DarkNet [22]}

Fig. 6. The structure of the COVIDNet architecture

DarkNet may be a convolutional neural network that's used as the backbone of YOLO (You solely look once). This technique has a progressive design designed for object detection. In DarkNet shown in Fig. 7. Every layer has one convolutional layer followed by BatchNorm, and LeakyReLU operations, while every $3 \times$ Conv layer has a similar setup 3 times in serial kind. The batch normalization operation is employed to standardize the inputs. Tabel I, shows the strongest and weakness points of DarkNet model.

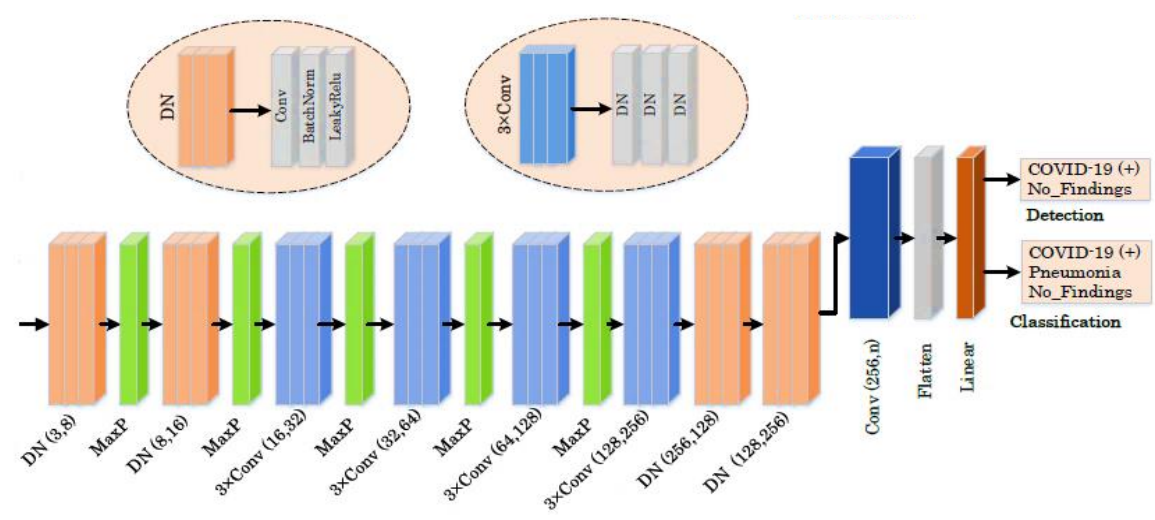

Fig. 7. The structure of the DarkNet architecture [22] 
LeakyReLU may be a variant of the ReLU operation accustomed to stop dying neurons. In contrast to ReLU or sigmoid activation functions, which have zero values within the negative part of their derivatives, LeakyReLU incorporates a little epsilon value to beat the dying neuron problem. The same as the Darknet-19 model, Maxpool methodology is employed all told the pooling operations. Maxpool downsizes AN input by taking the utmost of vicinity determined by its filter [23].

TABLE I. Advantage and Disadvantage of the six models(Related Works)

\begin{tabular}{|c|c|c|}
\hline Model & Advantages & Disadvantages \\
\hline Bayesian [9] & 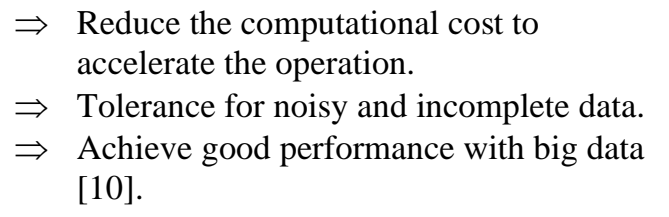 & $\begin{array}{l}\Rightarrow \text { Curse of dimensionality. } \\
\Rightarrow \text { Unavailability of highly efficient } \\
\text { performance. } \\
\Rightarrow \text { Time-consuming operations [11]. }\end{array}$ \\
\hline DenseNet [12] & $\begin{array}{l}\Rightarrow \text { Improved data flow through the network. } \\
\Rightarrow \text { Reduced the vanishing gradient } \\
\text { problems. } \\
\Rightarrow \text { Enhanced feature reuse and parameter } \\
\text { efficiency. } \\
\Rightarrow \text { Each layer provides a collective } \\
\text { knowledge of the network. } \\
\Rightarrow \text { That dense connection reduced over- } \\
\text { fitting [13]. }\end{array}$ & $\begin{array}{l}\Rightarrow \text { Very expensive in memory } \\
\text { consumption during training. } \\
\Rightarrow \text { Complexity. }\end{array}$ \\
\hline VGG [14] & $\begin{array}{l}\Rightarrow \text { Perform well with fewer parameters. } \\
\Rightarrow \text { Two ReLU layers instead of a single } \\
\text { ReLU layer. } \\
\Rightarrow \text { It's characterized by simplicity. } \\
\Rightarrow \text { Depth of the network provides more } \\
\text { complex features and lower cost. }\end{array}$ & $\begin{array}{l}\Rightarrow \text { Need for a long training duration. } \\
\Rightarrow \quad \text { Vanishing gradient problem [16]. }\end{array}$ \\
\hline ResNet [17] & $\begin{array}{l}\Rightarrow \text { Architecture does not need to fire all } \\
\text { neurons in each epoch. } \\
\Rightarrow \begin{array}{l}\text { Shortest training time and best accuracy } \\
\text { and performance. }\end{array} \\
\Rightarrow \text { Learnable approach [19]. }\end{array}$ & $\begin{array}{ll}\Rightarrow & \text { High computation time. } \\
\Rightarrow & \text { Implementation of Batch } \\
& \text { normalization layers }\end{array}$ \\
\hline COVIDNet [20] & $\begin{array}{l}\Rightarrow \text { High computational efficiency. } \\
\Rightarrow \text { High representational capacity [21]. } \\
\Rightarrow \text { Fine-grained design patterns rapidly and } \\
\text { automatically discovered. }\end{array}$ & $\begin{array}{l}\Rightarrow \text { Difficult and time-consuming design } \\
\text { patterns. }\end{array}$ \\
\hline DarkNet [22] & 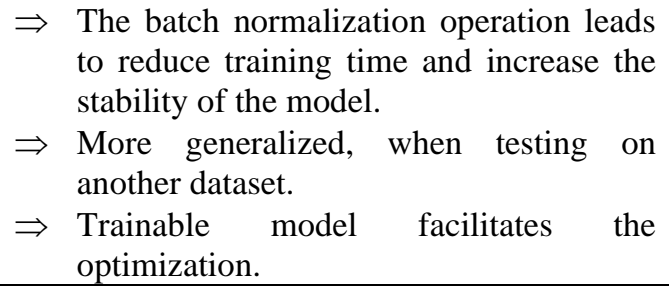 & $\Rightarrow$ Each grid can only detect one object. \\
\hline
\end{tabular}




\section{EXPERIMENTAL RESULTS}

Several steps followed for comparing the six CNN models to avoid bias, as shown in Figure. 8. As an initial step, for each of the methods mentioned in Sec II, we validated their results on each model's datasets either on CT scans like Bayesian, VGG, and COVIDNet or on X-Ray like ResNet, DenseNet, and DarkNet. This step is critical to assure achieving the best tuning for each model parameters. In the second step, one dataset was selected from each of the three CT datasets and the three models which works on CT images were trained and tested on this single dataset. Similarly, we selected a single X-ray dataset to validate and test models that works on X-ray images. The datasets are selected due to length and generality as discussed in Sec III.A. In the last step, all the models were trained and validated on the selected CT dataset, and then validated and trained on selected X-ray dataset.

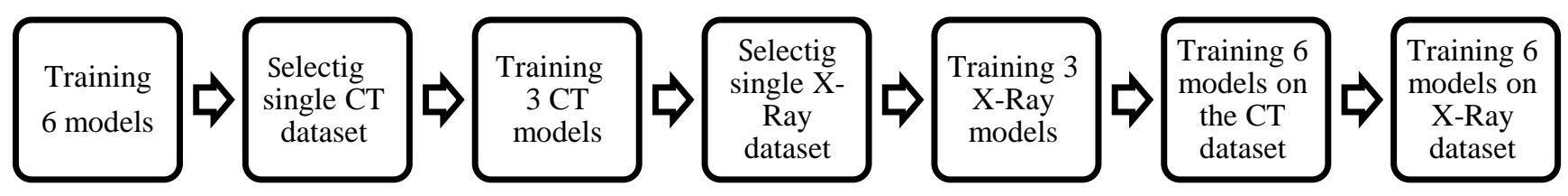

Fig. 8. Experimental results process flow

\section{A. Dataset Description}

The public datasets are used, that contains images of positive, or suspected COVID-19 infection. The primary dataset accustomed train and valuate models works on X-ray images is called COVIDx [24], it consists of 16,756 chest radiography images for 13,645 cases. Fig. 9. shows X-ray image samples from the COVIDx dataset with different infections.

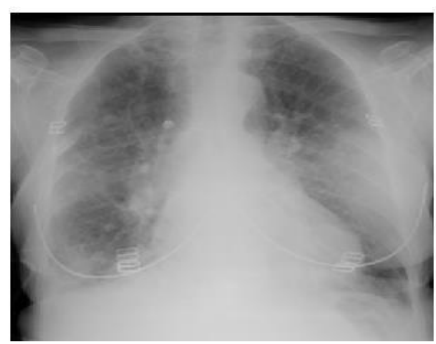

(a)

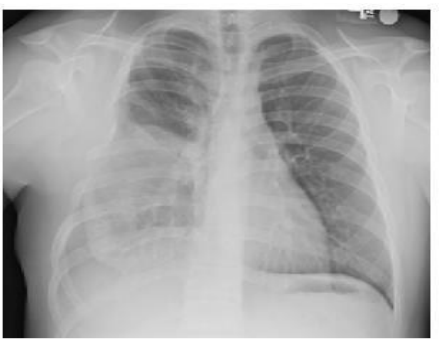

(b)

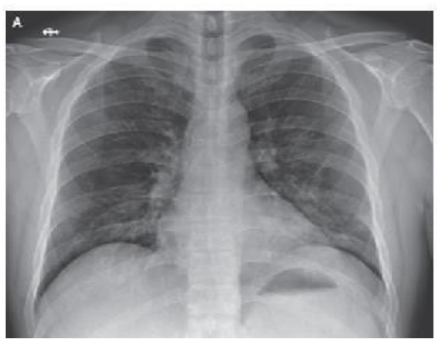

(c)

Fig. 9. Samples of COVIDx dataset, showing (a) COVID-19 case, (b) pneumonia cases, and (c) uninfected case [24]

The second dataset chosen for CT images consists of 104,009 chest CT images representing a 1,489 case. This information from hospital all over China as a part of the China association of Chest CT Image Investigation (CCCCII) [25]. Its images contain chest CT volumes across three different types of infection: COVID-19 case, pneumonia case, and uninfected case. Fig. 10. shows CT image samples from the COVIDx-CT dataset with different infections. For COVID-19 and pneumonia scans volumes, slices marked as containing lung abnormalities were indicated, as shown in the figures. We empirically split these two datasets to $60 \%$ as training, $20 \%$ as validation, and $20 \%$ as testing set, which showed the most accurate performance of all the applied models. Both CT and X-rays dataset have been selected according to size and generality. 


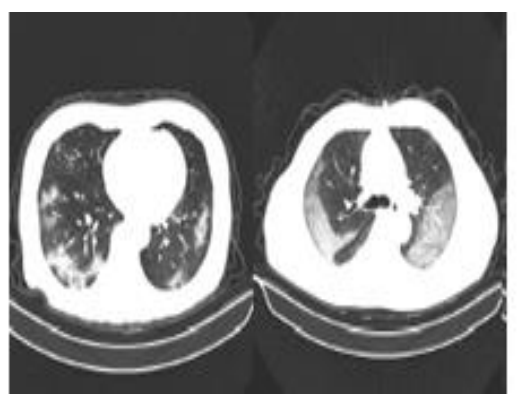

(a)

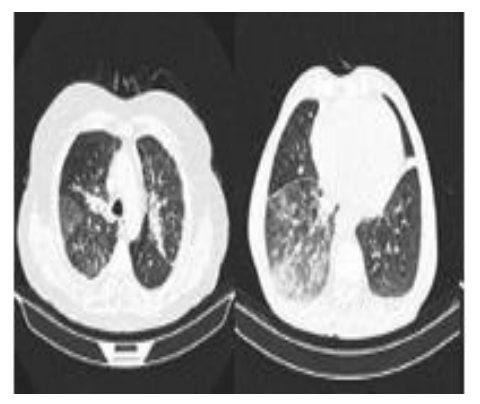

(b)

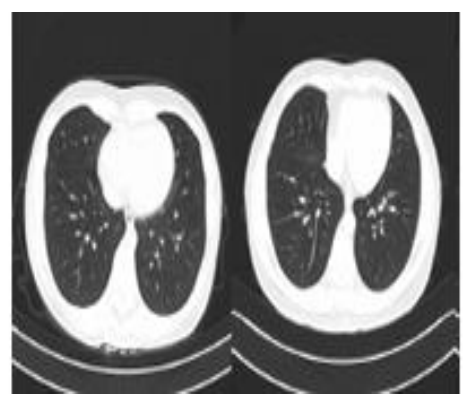

(c)

Fig. 10. Samples of COVID CT dataset, showing: (a) COVID-19 case, (b) pneumonia cases, and (c) uninfected case [25]

\section{B. Evaluation Metrics}

We take into account the following most common five metrics used in detection to evaluate each algorithm:

- Precision: The ratio of correctly predicted positive COVID19 patients to the total positive predictions (i.e. True positives and false positives). This metric gives ability of an algorithm determining the rate of false positives. The high the precision is the low the false positives are.

$$
\text { Precision }=\text { correct } /(\text { correct }+ \text { false })
$$

- Recall: is also known as the sensitivity of the algorithm. It is the ratio of correctly predicted positive outcomes (i.e. True positives) to the actual class observations (i.e. True positives and False negatives).

$$
\text { Recall }=\text { correct } /(\text { correct }+ \text { missed })
$$

- F1 Score: takes false positives and false negatives by taking the weighted average of the earlier mentioned metrics. F1 score is useful in cases where class distribution is uneven.

$$
F 1=2 *(\text { Precision } * \text { Recall }) /(\text { Precision }+ \text { Recall })
$$

- Accuracy: Is considered as the most used and intuitive measure in classification. It is defined as ratio of correct predictions to number of samples. Although high accuracy may be a good measure; however, it may not be the best in certain situations where the class distribution is not symmetric.

$$
\text { Accuracy }=(\text { True positives }+ \text { True negatives }) /(\text { Total samples })
$$

\section{Quantitative Evaluation}

We quantitatively assessed the performance and accuracy of the six discussed methods in the second. Secondly. The evaluation process was carried out in three steps. First, each model was trained and tested on its dataset according to the original paper for each model. This step is important to ensure that we achieve the best tuning of the various parameters and similar detection accuracy as reported in the publications of each model. Then we trained the three CT models using the same data together, as well as for the X-ray, and finally we trained all six models on one carefully selected dataset of each type (CT and X-ray). Table 2 and Table 3 show the quantitative results for each model using CT or X-ray dataset, respectively. The PC used in the experiment has 8 GB of RAM, with 11 GB of

\begin{tabular}{|c|c|c|c|c|c|c|}
\hline \multirow{2}{*}{ Model Name } & \multicolumn{6}{|c|}{ Information } \\
\hline & Data Type & Training & Validation & Test & Accuracy & Time \\
\hline Bayesian [26] & CT [26] & $57 \%$ & $16 \%$ & $27 \%$ & $71.4 \%$ & 8 hours \\
\hline VGG [27] & CT [27] & $60 \%$ & $20 \%$ & $20 \%$ & $96.1 \%$ & 6 hours \\
\hline COVIDNET [28] & CT [28] & $60 \%$ & $20 \%$ & $20 \%$ & $99 \%$ & 3 hours \\
\hline
\end{tabular}
GPU gtx 1080ti. We employed fixed batch size (refers to the number of training examples utilized in one iteration) of 60 .

TABLE II. THREE CT MODELS EACH ON ITS DATASET 
In table II. , Bayesian model ranks NonCOVID and COVID CT scan images. The model has an auto-encoder to extract the latent space options, and then by exploiting the latent space options, we have a tendency to have a readyto-use space unit to classify images by exploiting $\mathrm{K}$ nearest neighbors and Bayesian model. The VGG model implemented the Keras implementation, and used the performance to normalize the image information, using gradient descent optimizer. The COVIDNet model was trained via a random gradient drop with momentum, and a TensorFlow exploit, using Adam optimizer. To extend the dataset, we have employed augmentation techniques, thus for each image we perform: 1) cropping box overlap, 2) rotation, 3) horizontal, 4) vertical shears, 5) horizontal face, 6) density shift; 7) Expanding the batch rebalancing strategy. As can be clearly seen in Table II, COVID-net yields higher detection accuracy on CT images.

TABLE III. THREE X-RAY MODELS EACH ON ITS DATASET

\begin{tabular}{|c|c|c|c|c|c|c|}
\hline \multirow{2}{*}{ Model Name } & \multicolumn{6}{|c|}{ Information } \\
\hline & Data Type & Training & Validation & Test & Accuracy & Time \\
\hline DenseNet [29] & X-ray [29] & $60 \%$ & $20 \%$ & $20 \%$ & $82 \%$ & 5 hours \\
\hline DarkNet [30] & X-ray [30] & $70 \%$ & $20 \%$ & $10 \%$ & $84 \%$ & 7 hours \\
\hline ResNet [31] & X-ray [31] & $70 \%$ & $20 \%$ & $10 \%$ & $95.5 \%$ & 4 hours \\
\hline
\end{tabular}

In TABLE. III., DenseNet was trained on Keras and Tourch using the model DenseNet 121, which consist of 121 layer with Adam optimizer. DarkNet model was designed with both less layers, and filters in comparison to initial DarkNet, as it only have 17 layers instead of 19 layers, with gradient descent optimizer. ResNet model trained on Keras with the version of 50 layers and using Adam optimizer.

According to Table III, ResNet yields high detection accuracy with $95.5 \%$ with shorter training times. This privilege comes as a result to extended depth of the network.

In the second step, we tend to unify the dataset for similar models. We employed the following rates to data split data split (60\% training, 20 validations and $20 \%$ test). Models works on CT images trained and evaluated on the COVIDx-CT dataset. Equally, models works on X-ray have trained and evaluated on the COVIDx dataset. TABLE IV and TABLE V show the accuracy of these models throughout a unified dataset for each set of models according to type of images it works on, either CT or X-ray.

TABLE IV. THREE CT MODELS ON COVIDX-CT DATASET

\begin{tabular}{|c|c|c|c|c|c|}
\hline \multirow{2}{*}{ Model Name } & \multicolumn{5}{|c|}{ Information } \\
\hline & Precision & Recall & $f 1$ & Accuracy & Time \\
\hline Bayesian & 0.90 & 0.71 & 0.79 & $78 \%$ & 6 hours \\
\hline VGG & 0.90 & 0.90 & 0.90 & $94 \%$ & 4 hours \\
\hline COVID-Net & 0.98 & 0.97 & 0.99 & $99 \%$ & 3 hours \\
\hline
\end{tabular}

For the unified dataset, the COVID-NET outperforms various approaches with 99\% accuracy, It achieve highest detection accuracy with shortest time, due ability to adapt to changing dynamic data dynamics and operational requirements. 
TABLE V. THREE X-RAY MODELS ON COVIDX DATASET

\begin{tabular}{|c|c|c|c|c|c|}
\hline \multirow{2}{*}{ Model Name } & \multicolumn{5}{|c|}{ Information } \\
\cline { 2 - 6 } & Precision & Recall & $\boldsymbol{f 1}$ & Accuracy & Time \\
\hline DenseNet & 0.82 & 0.79 & 0.80 & $80 \%$ & 3 hours \\
\hline DarkNet & 0.99 & 0.90 & 0.95 & $99 \%$ & 3 hours \\
\hline ResNet & 0.92 & 0.94 & 0.93 & $94 \%$ & 4 hours \\
\hline
\end{tabular}

With X-ray data set, the Darknet model achieves the best accuracy of $99 \%$ discovery, due to the effectiveness of the model to work on different data sets.

In the last step, it was important to test the performance of all models on a CT or x-rays dataset. Thus, all models have been trained and evaluated on the CT dataset as reported in VI. Similarly, all models have been trained and evaluated on the X-ray dataset as reported in TABLE VII.

TABLE VI. SIX CT MODELS ON COVIDX-CT DATASET

\begin{tabular}{|c|c|c|c|c|c|}
\hline \multirow{2}{*}{ Model Name } & \multicolumn{5}{|c|}{ Information } \\
\cline { 2 - 6 } & Precision & Recall & $\boldsymbol{f 1}$ & Accuracy & Time \\
\hline COVID-Net & 0.98 & 0.97 & 0.99 & $99 \%$ & 4 hours \\
\hline VGG & 0.90 & 0.90 & 0.90 & $94 \%$ & 5 hours \\
\hline ResNet & 0.92 & 0.94 & 0.93 & $94 \%$ & 6 hours \\
\hline Bayesian & 0.90 & 0.71 & 0.79 & $78 \%$ & 3 hours \\
\hline DarkNet & 0.99 & 0.90 & 0.95 & $99 \%$ & $60 \%$ \\
\hline DenseNet & 0.82 & 0.79 & 0.80 & hours \\
\hline
\end{tabular}

TABLE VII. SIX X-RAY MODELS ON COVIDX DATASET

\begin{tabular}{|c|c|c|c|c|c|}
\hline \multirow{2}{*}{ Model Name } & \multicolumn{5}{|c|}{ Information } \\
\cline { 2 - 6 } & Precision & Recall & $\boldsymbol{f 1}$ & accuracy & 4 Time \\
\hline COVID-Net & 0.96 & 0.96 & 0.95 & $94 \%$ & 5 hours \\
\hline VGG & 0.70 & 0.72 & 0.70 & $85 \%$ & 4 hours \\
\hline ResNet & 0.93 & 0.94 & 0.94 & $95 \%$ & 6 hours \\
\hline Bayesian & 0.70 & 0.65 & 0.69 & $70 \%$ & 3 hours \\
\hline DarkNet & 0.98 & 0.90 & 0.94 & $98 \%$ & $86 \%$ \\
\hline DenseNet & 0.82 & 0.80 & 0.84 & 5 hours \\
\hline
\end{tabular}

The foremost effective performance on CT dataset as shown in TABLE. VI was DarkNet in addition to COVIDNet with an accuracy rate of 99\% with some variation at intervals the values of PRECISION, RECALL, and F1, as it adapt to new dataset and has the best cost function. On the other hand, as shown in TABLE. VII it can be seen that DarkNet model yield highest detection accuracy with 98\% on X-ray dataset, and PRECISION 0.98, RECALL 0.9, and F1 0.94. COVID-Net comes second in terms of accuracy with $94 \%$. 
Fig. 11. Shows the confusion matrix of the models that archive high results (COVID-Net on the CT dataset and DarkNet on the X-Ray dataset).

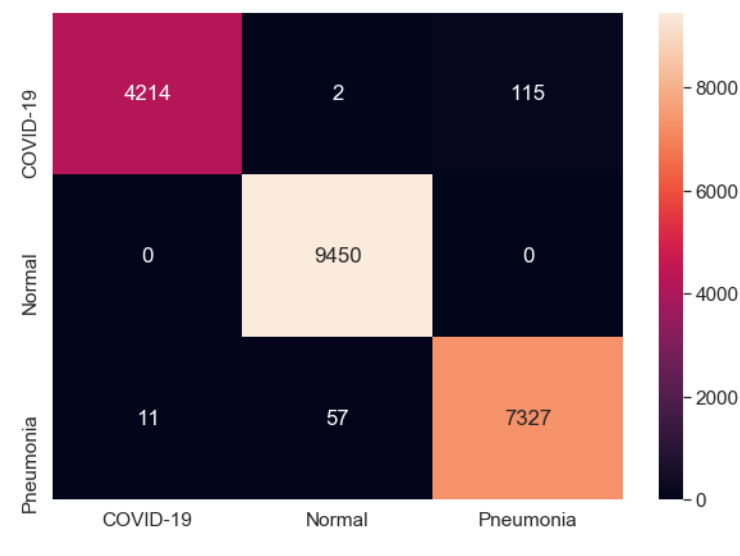

COVID-Net (CT)

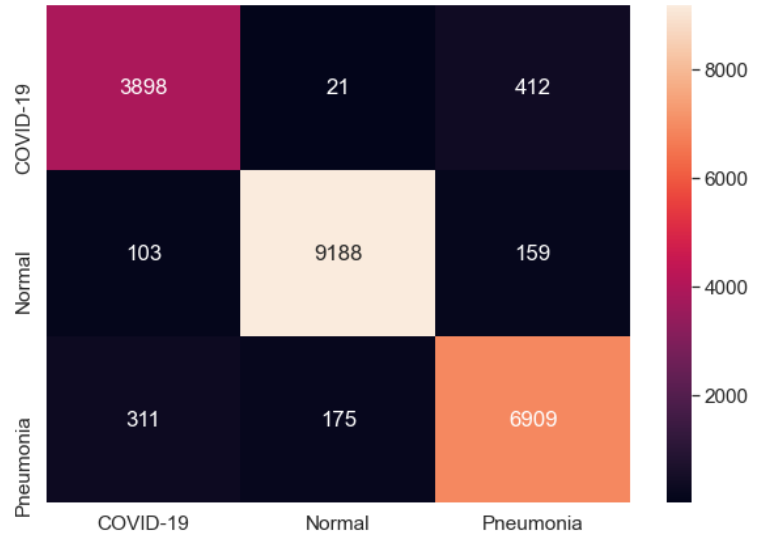

DarkNet (X-Ray)

Fig. 11. The confusion matrix of COVID-Net and DarkNet models

\section{CONCLUSION}

In recent days, medical images as X-ray and $\mathrm{CT}$ scans are a rapid diagnostic technique that has a key role in the treatment of COVID-19. In this study, we introduced the reason behind using X-rays and CT images in COVID-19 detection. Then, we described pre-trained CNN methods by using both images. We used an open public dataset of chest X-ray and CT images. We increased the data set by adding image augmentation methods. We proposed six pretrained deep CNN models such as the Bayesian, DenseNet, VGG, ResNet, COVIDNet, and DarkNet are used for transfer learning by using CT and x-ray images. The pre-trained DarkNet model provided the highest classification performance of automated COVID-19 classification with 99\% accuracy among the other five proposed models on the two types of dataset. While COVID-Net model provided high performance only on CT images with $99 \%$ accuracy.

\section{REFERENCES}

[1] H.Bai, B.Hsieh, Z.Xiong, K.Halsey, J.Choi, T.Tran and I.Pan. "Performance of Radiologists in Differentiating COVID-19 from Non-COVID-19 Viral Pneumonia at Chest CT". Radiology, Vol. 296, pp.E46-E54, 2020.

[2] K, Jeffrey. P. "Chest CT findings in 2019 novel coronavirus (2019-nCoV) infections from Wuhan, China: key points for the radiologist". In: Radiology; Vol. 295, 2020.

[3] N. Singh and J. Fratesi, "Chest ct imaging of an early canadian case of covid-19 in a 28-year-old man," CMAJ, vol. 192, pp. E455-E455, 2020.

[4] L.T.De Paolis, G.Aloisio "Augmented Reality in Minimally Invasive Surgery". Springer, Berlin, In: Mukhopadhyay S.C., Lay-Ekuakille A. (eds) Advances in Biomedical Sensing, Measurements, Instrumentation and Systems. Lecture Notes in Electrical Engineering, vol 55, 2010.

[5] M. Hammad, A. M. Iliyasu, A. Subasi, E. S. L. Ho and A. A. A. El-Latif, "A Multitier Deep Learning Model for Arrhythmia Detection," in IEEE Transactions on Instrumentation and Measurement, vol. 70, pp. 1-9, 2020.

[6] X. Xie, Z. Zhong, W. Zhao, C. Zheng, F. Wang, and J. Liu, "Chest ct for typical coronavirus disease 2019 (covid-19) pneumonia: Relationship to negative rt-pcr testing," Radiology, vol. 296, pp. E41-E45, 2020.

[7] A. Alghamdi and M. Hammad, "Detection of myocardial infarction based on novel deep transfer learning methods for urban healthcare in smart cities," springer, Vol. 1609, 2020.

[8] S.Pouyanfar, S.Sadiq, Y.Yan and H.Tian. A Survey on Deep Learning: Algorithms, Techniques, and Applications. ACM DL, Vol. 51, 2018.

[9] C.hang, Y.Han, F.Li, S.Gao, D.Song, H.Zhao, K.Fan and Y.Zhang. A New CNN-Bayesian Model for Extracting Improved Winter Wheat Spatial Distribution from GF-2 imagery. Remote Sensing, Vol. 11, pp.619, 2019.

[10] R.Chandradevan. Shallow Understanding on Bayesian Optimization. Medium. Available at: <https://towardsdatascience.com/shallow-understanding-on-bayesian-optimization-324b6c1f7083>, Vol. 18, 2021.

[11] L.Gao and Y.Ding. Disease prediction via Bayesian hyperparameter optimization and ensemble learning. BMC Research Notes, Vol. 13, 2020. 
[12] L.Sarker, M.M.Islam, T.Hannan, Z.Ahmed, "COVID-DenseNet: A Deep Learning Architecture to Detect COVID-19 from Chest Radiology Images". Preprints, Vol.151, 2020.

[13] G. Huang, Z. Liu, L. Van Der Maaten and K. Q. Weinberger, "Densely Connected Convolutional Networks," 2017 IEEE Conference on Computer Vision and Pattern Recognition (CVPR), Honolulu, pp. 2261-2269, 2017.

[14] S.Tammina "Transfer learning using VGG-16 with Deep Convolutional Neural Network for Classifying Images"; International Journal of Scientific and Research Publications (IJSRP). Vol. 9 pp. 2250-3153, 2019.

[15] H.Perez, J.Tah and A.Mosavi. Deep Learning for Detecting Building Defects Using Convolutional Neural Networks. Sensors, Vol. 19, pp.3556, 2019.

[16] M.D.Zeiler and R.Fergus (2014) Visualizing and Understanding Convolutional Networks. In: Fleet D., Pajdla T., Schiele B., Tuytelaars T. (eds) Computer Vision . Lecture Notes in Computer Science. Springer, Cham, vol 8689, pp. ECCV 2014. ECCV 2014.

[17] X. Li, L. Ding, L. Wang and F. Cao, "FPGA accelerates deep residual learning for image recognition," 2017 IEEE 2nd Information Technology, Networking, Electronic and Automation Control Conference (ITNEC), Chengdu, pp. 837-840, 2017.

[18] M. Hammad, "ResNet-Attention model for human authentication using ECG signals," Expert Systems, Vol $12547,2020$.

[19] Q.Zhang, C.Bai, Z.Liu, L.Yang, H.Yu, J.Zhao and H.Yuan. A GPU-based residual network for medical image classification in smart medicine. Information Sciences, Vol 536, pp.91-100, 2020.

[20] L.Wang, Z.Lin and A.Wong, COVID-Net: a tailored deep convolutional neural network design for detection of COVID-19 cases from chest X-ray images. Scientific Reports, 10(1), 2020.

[21] H.Gunraj, L.Wang and A.Wong. COVIDNet-CT: A Tailored Deep Convolutional Neural Network Design for Detection of COVID-19 Cases From Chest CT Images. Frontiers in Medicine, Vol 7, 2020.

[22] H C, D., An Overview of You Only Look Once: Unified, Real-Time Object Detection. International Journal for Research in Applied Science and Engineering Technology, Vol 8, pp.607-609, 2020.

[23] L.Barber and C.Budnick, Harder, Better, Faster, Stronger: Principles of Academic ProsperityHarder, Better, Faster, Stronger: Principles of Academic Prosperity. PsycCRITIQUES, Vol 5959, 2019.

[24] GitHub. Lindawangg/COVID-Net. [online] Available at: https://github.com/lindawangg/COVID-Net [Accessed 7 February 2021].

[25] GitHub. Haydengunraj/COVIDNet-CT. [online] Available at: https://github.com/haydengunraj/COVIDNet-CT [Accessed 7 February 2021].

[26] GitHub. Frankkramer-lab/covid19.MIScnn. [online] Available at: https://github.com/frankkramerlab/covid19.MIScnn [Accessed 7 February 2021].

[27] Kaggle.com. Starter_Keras_implementation. [online] Available at: https://www.kaggle.com/azaemon/starterkeras-implementation [Accessed 7 February 2021].

[28] GitHub. haydengunraj/COVIDNet-CT. [online] Available at: <https://github.com/haydengunraj/COVIDNetCT> [Accessed 7 February 2021].

[29] GitHub. Liuzhuang13/DenseNet. [online] Available at: https://github.com/liuzhuang13/DenseNet [Accessed 7 February 2021].

[30] GitHub. Muhammedtalo/COVID-19. [online] Available at: https://github.com/muhammedtalo/COVID-19 [Accessed 7 February 2021].

[31] GitHub. Stanleyjzheng/COVID-ResNet. [online] Available at: https://github.com/stanleyjzheng/COVID-ResNet [Accessed 7 February 2021]. 